\title{
Effects of stoichiometric doping in superconducting Bi-O-S compounds
}

\author{
Corentin Morice \\ E-mail: cm712@cam.ac.uk \\ Cavendish Laboratory, University of Cambridge, Cambridge CB3 0HE, United \\ Kingdom \\ Emilio Artacho \\ E-mail: ea245@cam.ac.uk \\ Cavendish Laboratory, University of Cambridge, Cambridge CB3 0HE, United \\ Kingdom \\ Nanogune and DIPC, Tolosa Hiribidea 76, 20018 San Sebastián, Spain \\ Basque Foundation for Science, Ikerbasque, 48011 Bilbao, Spain
}

\section{Sian E. Dutton}

Cavendish Laboratory, University of Cambridge, Cambridge CB3 0HE, United Kingdom

\author{
Daniel Molnar \\ Cavendish Laboratory, University of Cambridge, Cambridge CB3 0HE, United \\ Kingdom
}

\section{Hyeong-Jin Kim}

Cavendish Laboratory, University of Cambridge, Cambridge CB3 0HE, United Kingdom

\author{
Siddharth S. Saxena \\ E-mail: sss21@cam.ac.uk \\ Cavendish Laboratory, University of Cambridge, Cambridge CB3 0HE, United \\ Kingdom
}

\begin{abstract}
Newly discovered Bi-O-S compounds remain an enigma in attempts to understand their electronic properties. A recent study of $\mathrm{Bi}_{4} \mathrm{O}_{4} \mathrm{~S}_{3}$ has shown it to be a mixture of two phases, $\mathrm{Bi}_{2} \mathrm{OS}_{2}$ and $\mathrm{Bi}_{3} \mathrm{O}_{2} \mathrm{~S}_{3}$, the latter being superconducting [W. A. Phelan et al., J. Am. Chem. Soc. 135, 5372 (2013)]. Using density functional theory, we explore the electronic structure of both the phases and the effect of the introduction of stacking faults. Our results demonstrate that the $\mathrm{S}_{2}$ layers dope the bismuth-sulphur bands. The bands at the Fermi level are of clear two-dimensional character. One band manifold is confined to the two adjacent, square-lattice bismuth-sulphur planes,
\end{abstract}


a second manifold is confined to the square lattice of sulphur dimers. We show that the introduction of defects in the stacking does not influence the electronic structure. Finally, we also show that spin-orbit coupling does not have any significant effect on the states close to the Fermi level at the energy scale considered. 


\section{Introduction}

The recent discovery of superconductivity in two new compounds, $\mathrm{Bi}_{4} \mathrm{O}_{4} \mathrm{~S}_{3}[1,2,3,4]$ and $\mathrm{LaO}_{x} \mathrm{~F}_{1-x} \mathrm{BiS}_{2}[5,6,7,8]$, has raised great interest. All those compounds share the same structure: a stacking of alternating $\mathrm{BiS}_{2}$ bilayers and spacer layers. Superconductivity is thought to arise from doping of the $\mathrm{BiS}_{2}$ bilayers. Electron doping in $\mathrm{Sr}_{1-x} \mathrm{La}_{x} \mathrm{FBiS}_{2}$ [9], and $\mathrm{La}_{1-x} \mathrm{M}_{x} \mathrm{OBiS}_{2}(\mathrm{M}=\mathrm{Ti}, \mathrm{Zr}, \mathrm{Hf}, \mathrm{Th})$ [10] results in superconductivity. The versatility of the $\mathrm{La}(\mathrm{O}, \mathrm{F}) \mathrm{BiS}_{2}$ system has been demonstrated by replacement of $\mathrm{La}$ with a range of lanthanide $L n^{3+}$ ions $[11,12,13,14,15]$ - a maximum $T_{c}$ of $10.6 \mathrm{~K}$ is reported in $\mathrm{LaO}_{x} \mathrm{~F}_{1-x} \mathrm{BiS}_{2}$ for $x=0.5$.

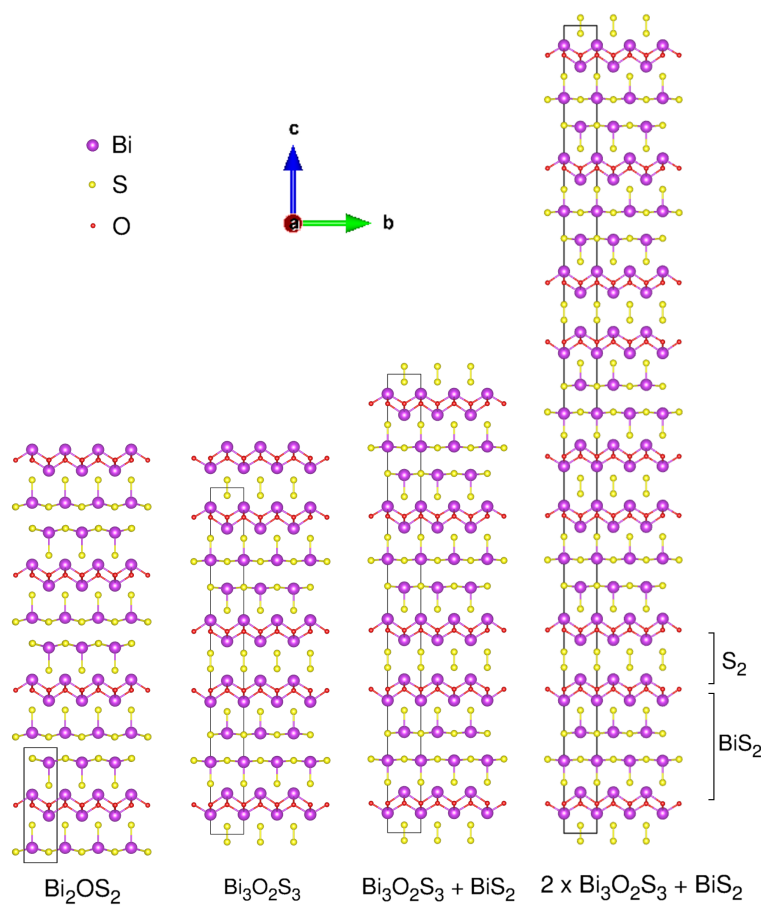

Figure 1. Crystal structure of $\mathrm{Bi}_{2} \mathrm{OS}_{2}, \mathrm{Bi}_{3} \mathrm{O}_{2} \mathrm{~S}_{3}, \mathrm{Bi}_{3} \mathrm{O}_{2} \mathrm{~S}_{3}$ with one stacking fault per unit cell of $\mathrm{Bi}_{3} \mathrm{O}_{2} \mathrm{~S}_{3}$, and $\mathrm{Bi}_{3} \mathrm{O}_{2} \mathrm{~S}_{3}$ with one stacking fault per two unit cell of $\mathrm{Bi}_{3} \mathrm{O}_{2} \mathrm{~S}_{3}$, represented with Vesta [16]. They are formed of a stacking of $\mathrm{BiS}_{2}, \mathrm{~S}_{2}$ and $\mathrm{Bi}_{2} \mathrm{O}_{2}$ layers, the latter being a spacer layer. These structures implement four different $\mathrm{S}_{2} / \mathrm{BiS}_{2}$ ratios : $0,1,2 / 3,4 / 5$, i.e. four different frequencies of occurrence of stacking faults. The black squares represent the unit cells used in the calculations.

Electronic structure calculations have mainly focused on the $\mathrm{LaO}_{x} \mathrm{~F}_{1-x} \mathrm{BiS}_{2}$ ( $\mathrm{x}$ $=0,0.5)$ materials partially due to uncertainties in the composition of the Bi-O-S superconducting phase $[17,18]$. These calculations indicated that the superconducting electrons are a mixture of Bismuth $6 p_{x, y}$ and Sulphur $3 p_{x, y}$ states $[17,18]$. These form 8 bands, four of which sit under the Fermi level, when the other four either are above the Fermi level or cross it. The electronic and thermodynamic properties of this group of materials are indeed exciting and enigmatic and suggestions range from spin-fluctuation 
mediated superconductivity [19] to proximity to ferroelectricity and charge density wave (CDW) instabilities [20, 21].

The coupling mechanism has been investigated in various ways. Electron-phonon interactions have been calculated in $\mathrm{La}(\mathrm{O}, \mathrm{F}) \mathrm{BiS}_{2}$, and yield a large electron-phonon coupling constant, suggesting superconductivity in this compound is strongly coupled and conventional [21, 20, 22]. Renormalisation-group calculations suggested triplet pairing and weak topological superconductivity [23, 24], a possibility studied in the context of quasiparticle interference [25]. Random phase approximation was applied to a two-orbital model [18], leading to an extended s-wave or d-wave pairing [19, 26].

The first superconductor of this family to be discovered, $\mathrm{Bi}_{4} \mathrm{O}_{4} \mathrm{~S}_{3}$, was studied in great detail $[27,28,29,30,31,32,33]$. Nevertheless, there were still doubts concerning its structure. However Phelan et al. recently published an extensive study of the chemistry of these compounds, and concluded that $\mathrm{Bi}_{4} \mathrm{O}_{4} \mathrm{~S}_{3}$ was actually a two-phased material [34]. The two phases are $\mathrm{Bi}_{2} \mathrm{OS}_{2}$ and $\mathrm{Bi}_{3} \mathrm{O}_{2} \mathrm{~S}_{3}$, the latter being assigned as the superconducting one. Single crystals of the superconducting phase have recently been synthesised [35].

Composed of $\mathrm{BiS}_{2}, \mathrm{~S}_{2}$ and $\mathrm{Bi}_{2} \mathrm{O}_{2}$ layers, the latter being a spacer layer (Figure 1), $\mathrm{Bi}_{3} \mathrm{O}_{2} \mathrm{~S}_{3}$ is made of alternating $\mathrm{BiS}_{2}$ bilayers and $\mathrm{S}_{2}$ layers, all separated by $\mathrm{Bi}_{2} \mathrm{O}_{2}$ spacer layers, whereas in $\mathrm{Bi}_{2} \mathrm{OS}_{2}$ only the two $\mathrm{BiS}_{2}$ bilayers are present. Here, we call BiS plane a two-dimensional squared lattice containing bismuth and sulphur atoms. We call $\mathrm{BiS}_{2}$ bilayer a structure containing two BiS planes and extra sulphurs localised on top or under each bismuth atom of the BiS planes. Figure 2 illustrates the difference between BiS plane and $\mathrm{BiS}_{2}$ bilayer.

Experimental results [34] suggest that superconductivity is suppressed by the inclusion of additional $\mathrm{BiS}_{2}$ bilayers disrupting the alternation of $\mathrm{S}_{2}$ and $\mathrm{BiS}_{2}$ sheets, which we call stacking faults. Phelan et al. studied these stacking faults in details, in particular displaying TEM data that shows without ambiguity the variations in stacking. Stacking faults play a significant role in superconducting properties of several families of materials [36]. For example, changes to the ground state have been observed in the presence of stacking faults in other systems, including modifications to superconductivity $[37,38]$.

In this paper we explore the electronic structure of the recently experimentally determined superconducting and non-superconducting phases of the Bi-O-S compounds. The role of introducing additional $\mathrm{BiS}_{2}$ bilayers in the superconductor $\mathrm{Bi}_{3} \mathrm{O}_{2} \mathrm{~S}_{3}$ is also investigated, as suggested by Phelan et al. in their experimental work [34]. Like in the $\mathrm{La}(\mathrm{O}, \mathrm{F}) \mathrm{BiS}_{2}$ systems we find that the $\mathrm{BiS}_{2}$ bilayers play a critical role in conduction. Our analysis indicates that the $\mathrm{S}_{2}$ sheets give rise to electron carriers in the bismuthsulphur bands. These electrons are localised within the BiS planes rather that through the $\mathrm{BiS}_{2}$ bilayer. Interruption of the $\mathrm{BiS}_{2}, \mathrm{~S}_{2}$ stacking sequence is shown not to influence the electronic structure. 


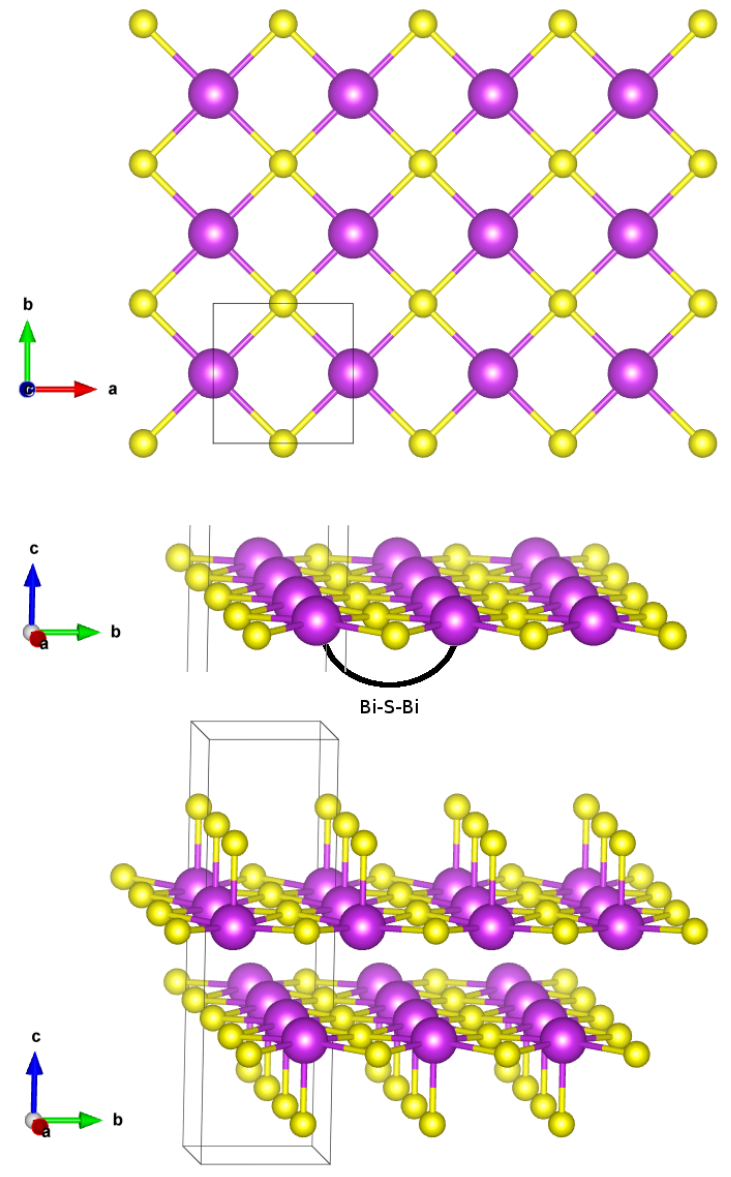

Figure 2. BiS plane seen from above (top), in perspective (middle), and $\mathrm{BiS}_{2}$ bilayer seen in perspective (bottom) represented with Vesta [16]. One $\mathrm{BiS}_{2}$ bilayer contains two BiS planes, and one extra S atom on top (or below) each Bi atom. The Bi-S-Bi angle is highlighted in the middle plot.

\section{Methods}

Band structures and density of states for $\mathrm{Bi}_{2} \mathrm{OS}_{2}$ and $\mathrm{Bi}_{3} \mathrm{O}_{2} \mathrm{~S}_{3}$ were calculated, using the SIESTA method [39, 40], implementing the generalized gradient approximation (GGA) in the shape of the Perdew, Burke, and Ernzerhof (PBE) functional. It uses normconserving pseudopotentials to replace the core electrons, while the valence electrons are described using atomic-like orbitals as basis states at the double zeta polarized level. In the case of the two known structures, experimentally determined structural parameters were used in our calculations [34] $\$$ Spin polarized calculations were found to

$\ddagger$ For the first phase, $\mathrm{Bi}_{2} \mathrm{OS}_{2}$, the space group is $\mathrm{P} 4 / \mathrm{nmm}$, and the lattice parameters are $a=3.9661$ $\AA$ and $c=13.798 \AA$. The atoms coordinates are Bi1 : $(0.25,0.25,0.59122), \mathrm{Bi} 2:(0.25,0.25,0.12989)$, $\mathrm{O}:(0.75,0.25,0.5), \mathrm{S} 1:(0.25,0.25,0.328), \mathrm{S} 2:(0.25,0.25,0.9063)$. For the second phase, $\mathrm{Bi}_{3} \mathrm{O}_{2} \mathrm{~S}_{3}$, the space group is $\mathrm{I} 4 / \mathrm{mmm}$, and the lattice parameters are $a=3.96721 \AA$ and $c=41.2847 \AA$. The atoms coordinates are Bi1 : (0,0,0.05699), Bi2: (0,0,0.20865), Bi3 : (0,0,0.38245), O : (0,0.5,0.0852), S1 : $(0,0,0.14595), \mathrm{S} 2:(0,0,0.2870), \mathrm{S} 3:(0,0,0.47748)$. All the occupancies are equal to one. 
be unnecessary. We used a k-point grid of $16 \times 16 \times 5$ for $\mathrm{Bi}_{2} \mathrm{OS}_{2}$, of $16 \times 16 \times 2$ for $\mathrm{Bi}_{3} \mathrm{O}_{2} \mathrm{~S}_{3}$ and of $16 \times 16 \times 1$ for the two other structures, after showing the electronic structure was well converged using them. We used an electronic temperature of $7 \mathrm{~K}$ and a real space mesh cutoff of 300 Ry for the real-space integration, necessary to calculate the matrix elements for some terms of the Hamiltonian. The convergence of these parameters was also checked.

In order to estimate the influence of spin-orbit coupling, we also performed all electron calculations on $\mathrm{Bi}_{3} \mathrm{O}_{2} \mathrm{~S}_{3}$ using full-potential linearised augmented plane waves, including spin-orbit coupling through a second-variational scheme, as implemented in the Elk program [41]. We used the PBE functional and the same k-point grid we used with SIESTA, along with a carefully converged high-quality set of parameters.

\section{Crystal structure}

The structures of the key materials for this study, $\mathrm{Bi}_{2} \mathrm{OS}_{2}$ and $\mathrm{Bi}_{3} \mathrm{O}_{2} \mathrm{~S}_{3}$, are taken from the experiments in [34]. In order to simulate how stacking faults perturb the electronic structure, we also investigated materials where the relative number of $\mathrm{BiS}_{2}$ bilayers and $\mathrm{S}_{2}$ layers was changed, as suggested by experiment, in particular by TEM data [34]. We did not use relaxed structures in order to keep consistency with the structures directly measured with X-rays.

In $\mathrm{Bi}_{3} \mathrm{O}_{2} \mathrm{~S}_{3}$, the stacking is $2 \times \mathrm{BiS}_{2} / \mathrm{Bi}_{2} \mathrm{O}_{2} / \mathrm{S}_{2} / \mathrm{Bi}_{2} \mathrm{O}_{2} / 2 \times \mathrm{BiS}_{2} / \mathrm{Bi}_{2} \mathrm{O}_{2} /$ $\mathrm{S}_{2} / \mathrm{Bi}_{2} \mathrm{O}_{2}$ (Figure 1). The sequence is repeated twice because of symmetry: $\mathrm{S}_{2}$ layers shift the $\mathrm{Bi}_{2} \mathrm{O}_{2}$ layers next to them on the $\mathrm{x}$ and $\mathrm{y}$ axis. Therefore the unit cell has to be doubled so that the stacking of one cell on another is possible.

To modify the ratio of $\mathrm{S}_{2}$ layers over $\mathrm{BiS}_{2}$ bilayers, we added a $\mathrm{BiS}_{2}$ bilayer, with its corresponding $\mathrm{Bi}_{2} \mathrm{O}_{2}$ spacer layer, at the top of $\mathrm{Bi}_{3} \mathrm{O}_{2} \mathrm{~S}_{3}$. We therefore obtain the following stacking : $2 \times \mathrm{BiS}_{2} / \mathrm{Bi}_{2} \mathrm{O}_{2} / \mathrm{S}_{2} / \mathrm{Bi}_{2} \mathrm{O}_{2} / 2 \times \mathrm{BiS}_{2} / \mathrm{Bi}_{2} \mathrm{O}_{2} / \mathrm{S}_{2} / \mathrm{Bi}_{2} \mathrm{O}_{2}$ $/ 2 \times \mathrm{BiS}_{2} / \mathrm{Bi}_{2} \mathrm{O}_{2}$. This can be done because $\mathrm{BiS}_{2}$ bilayers, unlike $\mathrm{S}_{2}$, do not shift $\mathrm{Bi}_{2} \mathrm{O}_{2}$ layers laterally. The stacking sequence is therefore not disturbed by the added block. Similarly we also constructed a structure in which an additional $\mathrm{BiS}_{2}$ bilayer was included every two units cells. In total, we calculated the electronic structure of compounds with four different $\mathrm{S}_{2} / \mathrm{BiS}_{2}$ ratios: $0,1,2 / 3$ and $4 / 5$.

The structure of $\mathrm{BiS}_{2}$ bilayers is different in $\mathrm{Bi}_{2} \mathrm{OS}_{2}$ and $\mathrm{Bi}_{3} \mathrm{O}_{2} \mathrm{~S}_{3}$, the most noticeable difference being the Bi-S-Bi angle in the BiS planes (Figure 2), which is larger in the parent phase. We thus have had to choose which $\mathrm{BiS}_{2}$ bilayer to add in the compounds with a modified stacking: $\mathrm{Bi}_{3} \mathrm{O}_{2} \mathrm{~S}_{3}+\mathrm{BiS}_{2}$ and $2 \mathrm{xBi}_{3} \mathrm{O}_{2} \mathrm{~S}_{3}+\mathrm{BiS}_{2}$ (Figure 1). We did calculations for both, and the results were extremely similar (e.g. the bands are less than $0.1 \mathrm{eV}$ apart). The results discussed below are the ones for $\mathrm{Bi}_{3} \mathrm{O}_{2} \mathrm{~S}_{3}$ 's $\mathrm{BiS}_{2}$ bilayers, as the stacking faults appear in this compound. 


\section{Electronic structure}

The band structures calculated with Elk and SIESTA are in very close agreement (Figure 3 , right hand side). The influence of spin-orbit coupling is very limited, with a difference under $0.15 \mathrm{eV}$ between energy levels with and without spin-orbit coupling (Figure 3, left hand side). The presence or absence of spin-orbit coupling has no impact on any of the features of the electronic structure discussed below. In the following we concentrate on electronic structures calculated with SIESTA.
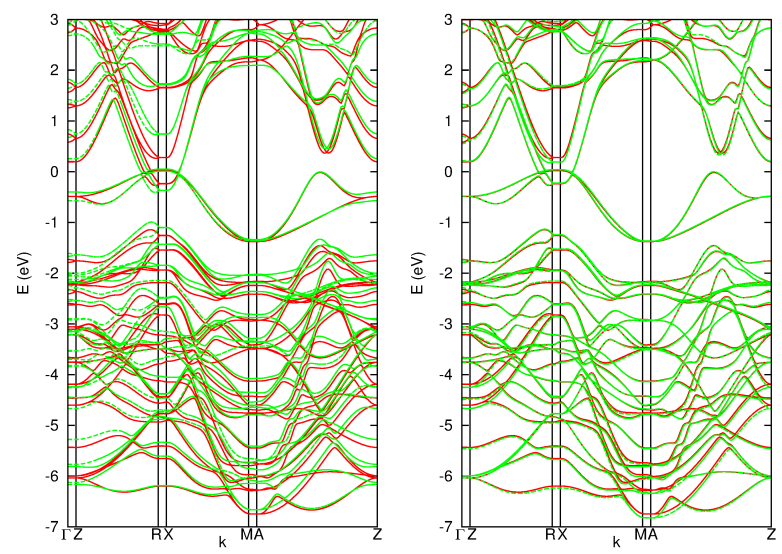

Figure 3. Left: band structure of $\mathrm{Bi}_{3} \mathrm{O}_{2} \mathrm{~S}_{3}$ calculated with Elk, with (red) and without (green) spin-orbit coupling. Right: band structure of $\mathrm{Bi}_{3} \mathrm{O}_{2} \mathrm{~S}_{3}$ calculated with Elk (red) and with SIESTA (green) without spin-orbit coupling. In all band structures presented in this paper, the Fermi level is at zero energy.

\subsection{Band structures}

$\mathrm{Bi}_{2} \mathrm{OS}_{2}$ and $\mathrm{Bi}_{3} \mathrm{O}_{2} \mathrm{~S}_{3}$ only differ by the replacement in the second compound of one out of two $\mathrm{BiS}_{2}$ bilayer by an $\mathrm{S}_{2}$ layer. In terms of stoichiometry, as noted by Phelan et al. [34], $\mathrm{Bi}_{2} \mathrm{OS}_{2}$ can be written $\mathrm{BiOBiS}_{2}$, just as the parent phase of the lanthanum compound, $\mathrm{LaOBiS}_{2}$.

The band structure of $\mathrm{Bi}_{2} \mathrm{OS}_{2}$ (Figure 4) has features close to the Fermi energy which are similar to those of the undoped $\mathrm{LaOBiS}_{2}$ phase $[17,18]$. For all band structures calculated, of specific interest is the set of bands crossing or just above the Fermi level near the $\mathrm{R}$ and $\mathrm{X}$ points, and approaching the Fermi level between the $\mathrm{A}$ and $\mathrm{Z}$ points (Figure 4). These were also found in $\mathrm{LaO}_{x} \mathrm{~F}_{1-x} \mathrm{BiS}_{2}[17,18]$, and were shown in these compounds to be composed of bismuth $6 p_{x, y}$ and sulphur $3 p_{x, y}$ orbitals. Partial density of states calculations enabled us to confirm that in all the compounds considered in this study these bands are composed of bismuth $6 p_{x, y}$ and sulphur $3 p_{x, y}$ orbitals, confirming the assignment done in [18] (Figure 4).

In the first case, $\mathrm{Bi}_{2} \mathrm{OS}_{2}$, these $\mathrm{BiS}$ bands are just above the Fermi level, at around $0.3 \mathrm{eV}$ at their minimum. This is very close to the corresponding minimum in $\mathrm{LaOBiS}_{2}$ 

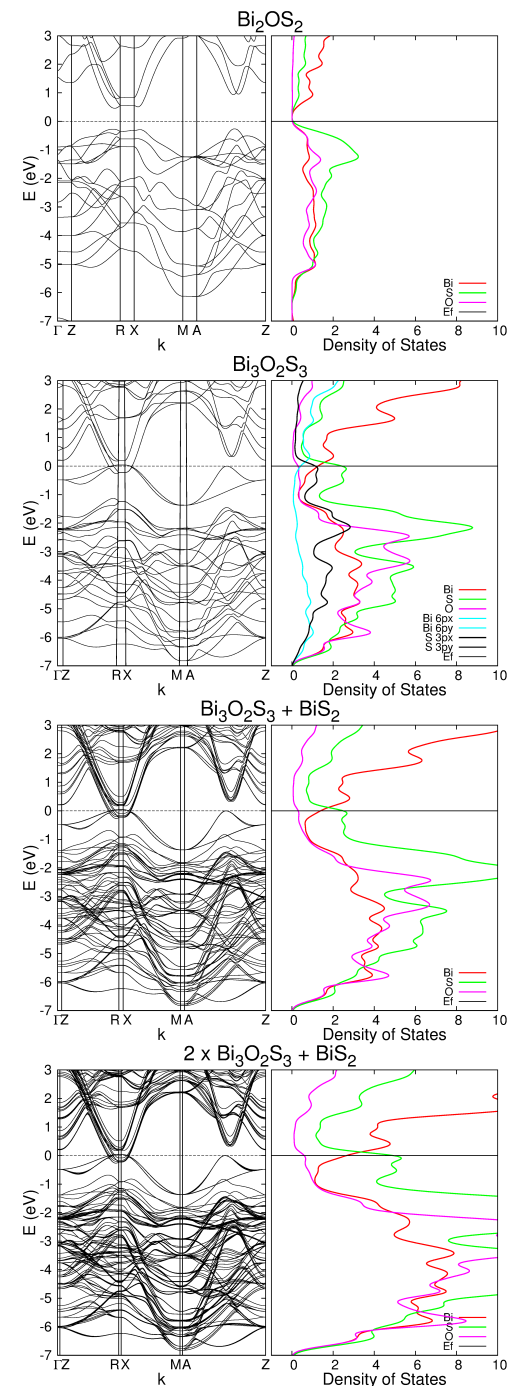

Figure 4. Band structures and densities of states projected onto the basis orbitals for Bi (red), S (green) and O (lilac) for the four simulated compounds. From top to bottom, they correspond to the structures from left to right in Figure 1. The two last compounds are simulations of stacking faults for two different frequencies in $\mathrm{Bi}_{3} \mathrm{O}_{2} \mathrm{~S}_{3}$. In $\mathrm{Bi}_{3} \mathrm{O}_{2} \mathrm{~S}_{3}$ without stacking faults, we plot the partial density of states for the bismuth $6 p_{x, y}$ (light blue) and sulphur $3 p_{x, y}$ orbitals (black). In each case, the $p_{x}$ and $p_{y}$ orbitals are indistinguishable. At the Fermi level, they each contribute approximately half of the density of state of the corresponding specie. These projections have been omitted in the other plots for clarity. The y-axis has been chosen so that the Fermi level is at zero energy.

$(0.2 \mathrm{eV})[17,18]$. The gap is $0.7 \mathrm{eV}$ wide, twice as large as in the $\mathrm{LaOBiS}_{2}$ case $(0.37$ eV) $[17,18]$.

In $\mathrm{Bi}_{3} \mathrm{O}_{2} \mathrm{~S}_{3}$, one of these $\mathrm{BiS}$ bands crosses the Fermi level near the $\mathrm{R}$ and $\mathrm{X}$ points, just as in $\mathrm{LaO}_{0.5} \mathrm{~F}_{0.5} \mathrm{BiS}_{2}[17,18]$. At these points it interacts with two other bands. These are in great majority composed of $\mathrm{S} 3 p$ states, as the projected density of states shows ("projected" refers to decomposing the total density of states in contributions 
from the different basis orbitals of the different atoms). These bands are not present in the parent phase, $\mathrm{Bi}_{2} \mathrm{OS}_{2}$; they come from the $\mathrm{S}_{2}$ layer. These two bands therefore correspond to the partly filled $\pi$ antibonding states in the $\mathrm{S}_{2}$ dimers. If fully occupied, this would formally correspond to $\mathrm{S}_{2}^{2-}$ dimers.

\subsection{Local densities of states}

To confirm these bands are coming from the $\mathrm{S}_{2}$ layer, we plotted the local density of states ("local" refers to decomposing the total density of states into real space), integrated in the range of energies between $-1.5 \mathrm{eV}$ and $0 \mathrm{eV}$ (Figure 5). The chosen range of energies contains the bands we are interested in.

The results are quite clear: it is indeed the $\mathrm{S}_{2}$ layer that gathers all the electron density. Moreover, this density is organised as a torus oriented along the $\mathrm{z}$ axis around each $\mathrm{S}$ atom (the fact that the density decreases near the atom comes from the pseudopotentials), which shows that the orbitals lying at or just above the Fermi level correspond mainly to $p_{x}, p_{y}$ electrons on the $\mathrm{S}_{2}$ dimers (oriented along $\mathrm{z}$ ). The concentration of the electron density on the atoms rather than on the S-S bond could be indicative of a weakening of the S-S interaction.

We also plotted the local density of states in real space for the range of energies between $0 \mathrm{eV}$ and $1.5 \mathrm{eV}$ (Figure 5). This range contains the characteristic bands present in all the $\mathrm{BiS}_{2}$-based compounds. We can clearly see that the charges are localised in the $\mathrm{BiS}_{2}$ bilayer, more precisely in the two BiS planes. The $\mathrm{S}$ atoms in the $\mathrm{BiS}_{2}$ bilayer which are outside the BiS planes gather as few electrons in that range of energy as the $\mathrm{S}$ atoms in the $\mathrm{S}_{2}$ layer.

\subsection{Pinning of the Fermi level}

In the band structures of $\mathrm{Bi}_{3} \mathrm{O}_{2} \mathrm{~S}_{3}$ with and without stacking faults, we observe that the top $\mathrm{S}_{2}$ band touches the Fermi level, without crossing it, approximately at equal distance from A and Z. Interestingly, this band is perfectly flat along the $z$ direction: this $\mathrm{S}_{2}$ related band has very marked two-dimensional character, and therefore it shows the characteristic discontinuous 2D Van Hove singularity (very clear at the Fermi level in the three lower panels of Fig. 4), which seems to be pinning the Fermi level. Such a discontinuity is suggestive of possible instabilities along the $(1,1,0)$ direction.

\subsection{Influence of stacking faults}

The electronic structure of $\mathrm{Bi}_{3} \mathrm{O}_{2} \mathrm{~S}_{3}$ with an added stacking fault is very close to that of $\mathrm{Bi}_{3} \mathrm{O}_{2} \mathrm{~S}_{3}$ (Figure 4). The only difference is that some bands below the $\mathrm{S}_{2}$ bands are raised slightly. It has strictly no impact on the bands close to the Fermi level.

\section{Conclusions}

The main conclusions of this study are: 


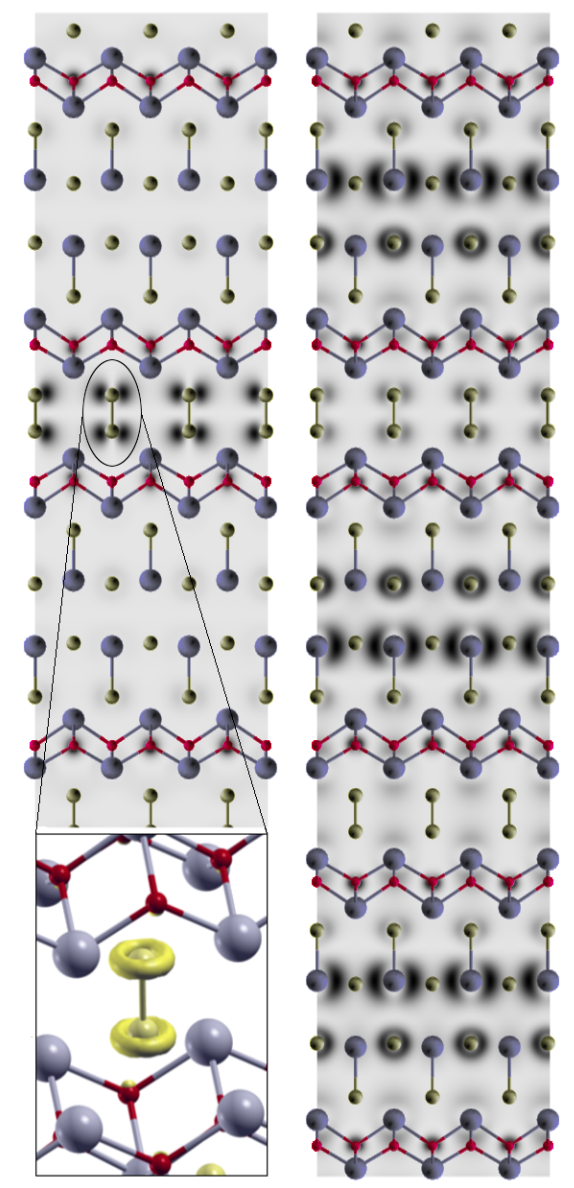

Figure 5. Local density of states of $\mathrm{Bi}_{3} \mathrm{O}_{2} \mathrm{~S}_{3}$ in real space integrated in the energy ranges [-1.5,0] (a) and $[0,1.5]$ (b), plotted with XCrySDen [42]. Framed is a 3D plot of an isosurface centred on the S-S dimer. In the first case, the $\mathrm{S}_{2}$ layer gathers most of the density. In the second case it is the BiS planes, without the extra $\mathrm{S}$ atoms, that gather the density. Half of the atoms represented are not in the plane corresponding to the density plot.

(i) The key result of this study is that, in spite of it being a nominally stoichiometric composition (no doping), the $\mathrm{S}_{2}$ layers in the $\mathrm{Bi}_{3} \mathrm{O}_{2} \mathrm{~S}_{3}$ compound act as effective dopants of the parent insulating compound. They push a $p_{x}, p_{y}$-like band of the $\mathrm{S}_{2}$ layer onto the bottom of the conduction band, thereby transferring part of the electron density from the $\mathrm{S}_{2}$ dimers to the BiS planes. This doping is in agreement with experiments, that show that $\mathrm{Bi}_{3} \mathrm{O}_{2} \mathrm{~S}_{3}$ is metallic [34].

Furthermore, the experimental observation of an anomalously short $\mathrm{S}-\mathrm{S}$ distance in the $\mathrm{S}_{2}$ layers is also consistent with electron depletion of the $\mathrm{S}_{2}$ layer. Indeed, a substantial residual force is observed for the $\mathrm{S}$ atoms in the $\mathrm{S}_{2}$ layer in the direction of elongating the distance, which suggests that the real system has a larger depletion of electrons from the $\mathrm{S}_{2}$ bands than the one obtained in the calculations.

(ii) The electronic structure close to the Fermi level is not modified by stacking faults. This indicates that the changes in the superconducting state caused by these are not 
related to the electronic structure

(iii) Finally, the calculations of density of states in real space indicate that the BiS bands, shown to be central for superconductivity in these compounds [18], actually come from the BiS planes. Therefore finding other structures having such planes would be very interesting in order to tune their interactions differently, and maybe transform their behaviour.

In summary, we studied in detail the electronic structure of the newly discovered superconductor $\mathrm{Bi}_{3} \mathrm{O}_{2} \mathrm{~S}_{3}$. We find the bismuth-sulphur bands corresponding to the $\mathrm{BiS}$ planes are doped by the $\mathrm{S}_{2}$ dimers, unlike the other superconducting members of the $\mathrm{BiS}_{2}$ family which are non-stoichiometric. We studied the influence of stacking faults on this material and found it does not influence the electronic structure. Finally, the fact that the electrons responsible for superconductivity are localised in the 2D bands of BiS planes suggests the possibility of other superconductors with BiS structural units.

\section{Acknowledgements}

We would like to thank Daniel Sanchez-Portal for his help with particular features of SIESTA, Arman Khojakhmetov for his exploratory work, and Gilbert G. Lonzarich, Richard Needs, Stephen Rowley, Sebastian Haines, Cheng Liu and Adrien Amigues for fruitful discussions. We acknowledge the support from EPSRC, Corpus Christi College, Darwin College, Jesus College, Cambridge Central Asia Forum, Cambridge Kazakhstan Development Trust and KAZATOMPROM. S.E.D would like to thank the Winton Program for the Physics of Sustainability. Part of this work was performed using the Darwin Supercomputer of the University of Cambridge High Performance Computing Service, using Strategic Research Infrastructure Funding from the Higher Education Funding Council for England and funding from the Science and Technology Facilities Council.

\section{References}

[1] Yoshikazu Mizuguchi, Hiroshi Fujihisa, Yoshito Gotoh, Katsuhiro Suzuki, Hidetomo Usui, Kazuhiko Kuroki, Satoshi Demura, Yoshihiko Takano, Hiroki Izawa, and Osuke Miura. BiS $2^{-}$ based layered superconductor $\mathrm{Bi}_{4} \mathrm{O}_{4} \mathrm{~S}_{3}$. Phys. Rev. B, 86:220510, 2012.

[2] Shiva Kumar Singh, Anuj Kumar, Bhasker Gahtori, Shruti, Gyaneshwar Sharma, Satyabrata Patnaik, and Veer P. S. Awana. Bulk superconductivity in bismuth oxysulfide $\mathrm{Bi}_{4} \mathrm{O}_{4} \mathrm{~S}_{3}$. Journal of the American Chemical Society, 134(40):16504-16507, 2012.

[3] Hiroshi Takatsu, Yoshikazu Mizuguchi, Hiroki Izawa, Osuke Miura, and Hiroaki Kadowaki. Bulk superconductivity in $\mathrm{Bi}_{4} \mathrm{O}_{4} \mathrm{~S}_{3}$ revealed by specific heat measurement. Journal of the Physical Society of Japan, 81(12):125002, 2012.

[4] S.G. Tan, L.J. Li, Y. Liu, P. Tong, B.C. Zhao, W.J. Lu, and Y.P. Sun. Superconducting and thermoelectric properties of new layered superconductor $\mathrm{Bi}_{4} \mathrm{O}_{4} \mathrm{~S}_{3}$. Physica C: Superconductivity, 483(0):94-96, 2012. 
[5] V.P.S. Awana, Anuj Kumar, Rajveer Jha, Shiva Kumar Singh, Anand Pal, Shruti, J. Saha, and S. Patnaik. Appearance of superconductivity in layered $\mathrm{LaO}_{0.5} \mathrm{~F}_{0.5} \mathrm{BiS}_{2}$. Solid State Communications, 157(0):21 - 23, 2013.

[6] K. Deguchi, Y. Mizuguchi, S. Demura, H. Hara, T. Watanabe, S. J. Denholme, M. Fujioka, H. Okazaki, T. Ozaki, H. Takeya, T. Yamaguchi, O. Miura, and Y. Takano. Evolution of superconductivity in $\mathrm{LaO}_{1-x} \mathrm{~F}_{x} \mathrm{BiS}_{2}$ prepared by high-pressure technique. EPL (Europhysics Letters), 101(1):17004, 2013.

[7] Hisashi Kotegawa, Yusuke Tomita, Hideki Tou, Hiroki Izawa, Yoshikazu Mizuguchi, Osuke Miura, Satoshi Demura, Keita Deguchi, and Yoshihiko Takano. Pressure study of $\mathrm{BiS}_{2}$ based superconductors $\mathrm{Bi}_{4} \mathrm{O}_{4} \mathrm{~S}_{3}$ and $\mathrm{La}(\mathrm{O}, \mathrm{F}) \mathrm{BiS}_{2}$. Journal of the Physical Society of Japan, 81(10):103702, 2012.

[8] Yoshikazu Mizuguchi, Satoshi Demura, Keita Deguchi, Yoshihiko Takano, Hiroshi Fujihisa, Yoshito Gotoh, Hiroki Izawa, and Osuke Miura. Superconductivity in novel $\mathrm{BiS}_{2}$-based layered superconductor $\mathrm{LaO}_{1-x} \mathrm{~F}_{x} \mathrm{BiS}_{2}$. Journal of the Physical Society of Japan, 81(11):114725, 2012.

[9] Xi Lin, Xinxin Ni, Bin Chen, Xiaofeng Xu, Xuxin Yang, Jianhui Dai, Yuke Li, Xiaojun Yang, Yongkang Luo, Qian Tao, Guanghan Cao, and Zhuan Xu. Superconductivity induced by la doping in $\mathrm{Sr}_{1-x} \mathrm{La}_{x} \mathrm{FBiS}_{2}$. Phys. Rev. B, 87:020504, 2013.

[10] D. Yazici, K. Huang, B. D. White, I. Jeon, V. W. Burnett, A. J. Friedman, I. K. Lum, M. Nallaiyan, S. Spagna, and M. B. Maple. Superconductivity induced by electron doping in $\mathrm{La}_{1-x} \mathrm{M}_{x} \mathrm{OBiS}_{2}$ (M= Ti, Zr, Hf, Th). Phys. Rev. B, 87(17):174512, 2013.

[11] Satoshi Demura, Yoshikazu Mizuguchi, Keita Deguchi, Hiroyuki Okazaki, Hiroshi Hara, Tohru Watanabe, Saleem James Denholme, Masaya Fujioka, Toshinori Ozaki, Hiroshi Fujihisa, Yoshito Gotoh, Osuke Miura, Takahide Yamaguchi, Hiroyuki Takeya, and Yoshihiko Takano. New member of $\mathrm{BiS}_{2}$-based superconductor $\mathrm{NdO}_{1-x} \mathrm{~F}_{x} \mathrm{BiS}_{2}$. Journal of the Physical Society of Japan, 82(3):033708, 2013.

[12] Rajveer Jha, Anuj Kumar, Shiva Kumar Singh, and V.P.S. Awana. Synthesis and superconductivity of new $\mathrm{BiS}_{2}$ based superconductor $\mathrm{PrO}_{0.5} \mathrm{~F}_{0.5} \mathrm{BiS}_{2}$. Journal of Superconductivity and Novel Magnetism, 26(3):499-502, 2013.

[13] Rajveer Jha, Anuj Kumar, Shiva Kumar Singh, and V. P. S. Awana. Superconductivity at $5 \mathrm{~K}$ in $\mathrm{NdO}_{0.5} \mathrm{~F}_{0.5} \mathrm{BiS}_{2}$. Journal of Applied Physics, 113(5):056102, 2013.

[14] Jie Xing, Sheng Li, Xiaxin Ding, Huan Yang, and Hai-Hu Wen. Superconductivity appears in the vicinity of semiconducting-like behavior in $\mathrm{CeO}_{1-x} \mathrm{~F}_{x} \mathrm{BiS}_{2}$. Phys. Rev. B, 86:214518, 2012.

[15] D. Yazici, K. Huang, B.D. White, A.H. Chang, A.J. Friedman, and M.B. Maple. Superconductivity of F-substituted $\mathrm{LnOBiS}_{2}$ ( $\left.\mathrm{Ln}=\mathrm{La}, \mathrm{Ce}, \mathrm{Pr}, \mathrm{Nd}, \mathrm{Yb}\right)$ compounds. Philosophical Magazine, 93(6):673-680, 2013.

[16] Koichi Momma and Fujio Izumi. VESTA3 for three-dimensional visualization of crystal, volumetric and morphology data. Journal of Applied Crystallography, 44(6):1272-1276, 2011.

[17] I.R. Shein and A.L. Ivanovskii. Electronic band structure and fermi surface for new layered superconductor $\mathrm{LaO}_{0.5} \mathrm{~F}_{0.5} \mathrm{BiS}_{2}$ in comparison with parent phase $\mathrm{LaOBiS}_{2}$ from first principles. JETP Letters, 96(12):769-774, 2013.

[18] Hidetomo Usui, Katsuhiro Suzuki, and Kazuhiko Kuroki. Minimal electronic models for superconducting $\mathrm{BiS}_{2}$ layers. Phys. Rev. B, 86:220501, 2012.

[19] G. B. Martins, A. Moreo, and E. Dagotto. RPA analysis of a two-orbital model for the $\mathrm{BiS}_{2}$-based superconductors. Phys. Rev. B, 87:081102, 2013.

[20] T. Yildirim. Ferroelectric soft phonons, charge density wave instability, and strong electron-phonon coupling in $\mathrm{BiS}_{2}$ layered superconductors: A first-principles study. Phys. Rev. B, 87:020506, 2013.

[21] Xiangang Wan, Hang-Chen Ding, Sergey Y. Savrasov, and Chun-Gang Duan. Electron-phonon superconductivity near charge-density-wave instability in $\mathrm{LaO}_{1-x} \mathrm{~F}_{x} \mathrm{BiS}_{2}$ : Density-functional calculations. Phys. Rev. B, 87:115124, 2013.

[22] B. Li, Z. W. Xing, and G. Q. Huang. Phonon spectra and superconductivity of the $\mathrm{BiS}_{2}$-based 
compounds $\mathrm{LaO}_{1-x} \mathrm{~F}_{x} \mathrm{BiS}_{2}$. EPL (Europhysics Letters), 101(4):47002, 2013.

[23] H. Yao and F. Yang. Topological Odd-Parity Superconductivity at Type-II 2D Van Hove Singularities. ArXiv e-prints, 2013.

[24] Yang Yang, Wan-Sheng Wang, Yuan-Yuan Xiang, Zheng-Zao Li, and Qiang-Hua Wang. Triplet pairing and possible weak topological superconductivity in bis-based superconductors. Phys. Rev. B, 88:094519, 2013.

[25] Yi Gao, Tao Zhou, Huaixiang Huang, Peiqing Tong, and Qiang-Hua Wang. Testing the $d_{x^{2}-y^{2}}^{*}$-wave pairing symmetry by quasiparticle interference and knight shift in $\mathrm{BiS}_{2}$-based superconductors. Phys. Rev. B, 90:054518, 2014.

[26] Tao Zhou and Z.D. Wang. Probing the superconducting pairing symmetry from spin excitations in $\mathrm{BiS}_{2}$ based superconductors. Journal of Superconductivity and Novel Magnetism, 26(8):2735$2740,2013$.

[27] P. Srivatsava, Shruti, and S. Patnaik. Structural, Electromagnetic, and Thermoelectric properties of $\mathrm{Bi}_{4} \mathrm{O}_{4} \mathrm{~S}_{3}$ Superconductor. ArXiv e-prints, 2013.

[28] Clastin I. Sathish, Hai Luke Feng, Youguo Shi, and Kazunari Yamaura. Superconductivity in bismuth oxysulfide $\mathrm{bi}_{4} \mathrm{O}_{4} \mathrm{~s}_{3}$. Journal of the Physical Society of Japan, 82(7):074703, 2013.

[29] Shruti, P. Srivastava, and S. Patnaik. Evidence for Fully Gapped Strong Coupling S-wave Superconductivity in $\mathrm{Bi}_{4} \mathrm{O}_{4} \mathrm{~S}_{3}$. ArXiv e-prints, 2013.

[30] Sheng Li, Huan Yang, DeLong Fang, ZhenYu Wang, Jian Tao, XiaXin Ding, and HaiHu Wen. Strong coupling superconductivity and prominent superconducting fluctuations in the new superconductor $\mathrm{Bi}_{4} \mathrm{O}_{4} \mathrm{~S}_{3}$. Science China Physics, Mechanics and Astronomy, 56(11):2019-2025, 2013.

[31] P. K. Biswas, A. Amato, C. Baines, R. Khasanov, H. Luetkens, Hechang Lei, C. Petrovic, and E. Morenzoni. Low superfluid density and possible multigap superconductivity in the $\mathrm{BiS}_{2}$-based layered superconductor $\mathrm{Bi}_{4} \mathrm{O}_{4} \mathrm{~S}_{3}$. Phys. Rev. B, 88:224515, 2013.

[32] G.K. Selvan, M. Kanagaraj, S. E. Muthu, R. Jha, V. P. S. Awana, and S. Arumugam.

[33] Rajveer Jha and V.P.S. Awana. Effect of se doping in recently discovered layered $\mathrm{Bi}_{4} \mathrm{O}_{4} \mathrm{~S}_{3}$ superconductor. Physica C: Superconductivity, 498(0):45 - 49, 2014.

[34] W. Adam Phelan, David C. Wallace, Kathryn E. Arpino, James R. Neilson, Kenneth J. Livi, Che R. Seabourne, Andrew J. Scott, and Tyrel M. McQueen. Stacking variants and superconductivity in the BiOS system. Journal of the American Chemical Society, 135(14):5372-5374, 2013.

[35] Jifeng Shao, Zhongheng Liu, Xiong Yao, Li Pi, Shun Tan, Changjin Zhang, and Yuheng Zhang. Bulk superconductivity in single-phase $\mathrm{Bi}_{3} \mathrm{O}_{2} \mathrm{~S}_{3}$. physica status solidi (RRL), Rapid Research Letters, 8(10):845-848, 2014.

[36] M. Weger. Explanation for the surface dependence of the tunneling gap in $\mathrm{Nb}_{3} \mathrm{Sn}$. Solid State Communications, 9(2):107 - 111, 1971.

[37] N. Banno, T. Takeuchi, K. Tsuchiya, K. Nakagawa, Y. Sakurai, K. Kurushima, and M. Saeda. Possible pining centers in transformation-processed $\mathrm{Nb}_{3} \mathrm{Al}$ superconductors. Applied Superconductivity, IEEE Transactions on, 22(3):6001504-6001504, 2012.

[38] U. Essmann and G. Zerweck. $1 / 2<111>$ stacking faults in A15 $(\beta$-w) superconductors. Physica Status Solidi (B), 57(2):611-616, 1973.

[39] Emilio Artacho, E Anglada, O Dieguez, J D Gale, A Garcia, J Junquera, R M Martin, P Ordejon, J M Pruneda, D Sanchez-Portal, and J M Soler. The SIESTA method; developments and applicability. Journal of Physics: Condensed Matter, 20(6):064208, 2008.

[40] Jose M Soler, Emilio Artacho, Julian D Gale, Alberto Garcia, Javier Junquera, Pablo Ordejon, and Daniel Sanchez-Portal. The SIESTA method for ab initio order- $\mathrm{n}$ materials simulation. Journal of Physics: Condensed Matter, 14(11):2745, 2002.

[41] http://elk.sourceforge.net/.

[42] Anton Kokalj. Computer graphics and graphical user interfaces as tools in simulations of matter at the atomic scale. Computational Materials Science, 28:155 - 168, 2003. 